\title{
Georges de Scudéry, Ibrahim ou l'Illustre Bassa
}

Fasano-Paris, Schena-Presses de l'Université de Paris-Sorbonne, 2 vols («Biblioteca della Ricerca», Testi stranieri 36)

\section{Madeleine Bertaud}

\section{Q OpenEdition}

\section{Journals}

\section{Édition électronique}

URL : http://journals.openedition.org/studifrancesi/32706

DOI : $10.4000 /$ studifrancesi.32706

ISSN : 2421-5856

Éditeur

Rosenberg \& Sellier

\section{Édition imprimée}

Date de publication : 1 décembre 2005

Pagination : 599-600

ISSN : 0039-2944

\section{Référence électronique}

Madeleine Bertaud, « Georges de Scudéry, Ibrahim ou I'Illustre Bassa », Studi Francesi [En ligne], 147 (XLX | III) | 2005, mis en ligne le 01 novembre 2015, consulté le 20 avril 2021. URL : http:// journals.openedition.org/studifrancesi/32706 ; DOI : https://doi.org/10.4000/studifrancesi.32706

Ce document a été généré automatiquement le 20 avril 2021.

\section{c) (†)}

Studi Francesi è distribuita con Licenza Creative Commons Attribuzione - Non commerciale - Non opere derivate 4.0 Internazionale. 


\section{Georges de Scudéry, Ibrahim ou l'Illustre Bassa}

Fasano-Paris, Schena-Presses de l'Université de Paris-Sorbonne, 2 vols («Biblioteca della Ricerca», Testi stranieri 36)

\section{Madeleine Bertaud}

\section{RÉFÉRENCE}

Georges de Scudéry, Ibrahim ou l'Illustre Bassa, Introduction et notes à l'Épitre et à la Préface par Rosa Galli Pellegrini. Établissement du texte, notes, annexes et fiches historiques par Antonella Arrigoni, Fasano-Paris, Schena-Presses de l'Université de Paris-Sorbonne, 2 vols («Biblioteca della Ricerca», Testi stranieri 36), 1228 pp.

1 Les importants travaux que Rosa Galli Pellegrini a consacrés à Georges de Scudéry poète, prosateur, romancier, sont bien connus ; on rappellera seulement son édition en deux volumes des Poésies diverses (1983-1984) et celle des Autres Euvres (1989). Par ailleurs, en 1999, Antonella Arrigoni a soutenu à l'Université de Turin une thèse de doctorat intitulée 'Ibrahim ou l'Illustre Bassa' di Georges de Scudéry. L'analisi delle fonti storiche e il problema critico dell'attribuzione. Aussi n'est-on pas surpris du travail à quatre mains qu'elles nous offrent avec cette édition savante d'Ibrahim, selon une formule que Rosa Galli Pellegrini avait déjà pratiquée avec bonheur il y a quelques années, en association avec Cristina Bernazzoli pour Alaric, ou Rome vaincue.

2 Les Scudéry, connus seulement, il y a un demi-siècle, d'une poignée d'érudits, ont aujourd'hui la faveur de la critique. En témoignent, pour ne citer qu'eux, la thèse d'Éveline Dutertre sur Scudéry dramaturge, le colloque du Havre organisé en 1991 par Alain Niderst, l'édition de la Clélie par Chantal Morlet-Chantalat, les études consacrées par Delphine Denis aux ouvrages de galanterie, et encore Le Grand Cyrus mis en ligne par l'équipe que dirige Claude Bourqui. Le moment était bien choisi pour rendre des lecteurs à Ibrahim, d'autant que la tragi-comédie du même titre, inspirée du roman, avait été rééditée par É. Dutertre à la STFM en 1998. L'entreprise vient à point; à ce seul 
titre, elle mérite d'être saluée. Le premier en date des grands romans scudéryens - 4 parties de 5 livres chacune - publié à Paris, chez A. de Sommaville entre 1641 et 1644, puis à Rouen, pour la Compagnie des Libraires du Palais en 1665, n'avait plus été réédité depuis 1723 (à Paris, chez $\mathrm{P}$. Witte). Or Ibrahim, qui a pour cadre principal le règne florissant de Soliman, dit "le Magnifique », est une pièce majeure dans l'importante production de «turqueries » qui marqua l'Âge classique.

3 L'Introduction de Rosa Galli Pellegrini, à la fois riche et concise, comprenant un résumé de ce roman de complexe structure, est un précieux guide pour le lecteur. Elle revient sur la question, toujours pendante, de l'auteur pour s'arrêter à Georges sans réduire à zéro la part de Madeleine, en soulignant scrupuleusement les limites de la critique d'attribution. Elle considère le roman dans son rapport avec ses sources historiques (en premier lieu, L'Histoire de la décadence de l'Empire grec de Chalcondyle, traduite par Blaise de Vigenère, avec la Continuation d'Artus Thomas), dont une partie est conservée, tandis qu'une autre est modifiée (la modification la plus remarquable étant qu'Ibrahim échappe à la mort alors que le favori de Soliman, victime de la jalousie de Roxelane, la "Sultane reine", ou peut-être suspecté d'entretenir des relations secrètes avec Charles-Quint, périt de mort violente en 1536), l'ensemble se mêlant aux éléments fictionnels : personnages et événements (le nom d'Isabelle Grimaldi n'est pas inventé, mais le personnage et l'intrigue amoureuse sont entièrement sortis de l'imagination de l'auteur). Cette utilisation des sources trouve son explication, R. Galli Pellegrini l'a bien compris, non seulement dans la technique du romancier et dans ses orientations littéraires, entre baroque et classicisme, mais aussi dans la politique étrangère de Richelieu - l'attachement de Georges de Scudéry au cardinal étant connu.

D'autres pages de cette introduction évoquent les salons, le goût mondain pour l'analyse du cœur, longuement développée au fil des lettres, des dialogues et des monologues, la composition par histoires entrelacées, qui tout en rappelant L'Astrée, est originale: parmi les histoires secondaires, les unes, orientales comme l'histoire principale, ont une thématique fortement tragique, tandis que les autres, occidentales (génoises pour la plupart), plus hétérogènes et pour certaines comiques (l'histoire du Marquis François), sont largement vouées à l'analyse des sentiments, au romanesque, à la mondanité.

5 Enfin, l'importance des avant-textes, l'Épître dédicatoire à Marguerite de Rohan, dans laquelle Georges de Scudéry désigne "son lecteur d'élection: un public de dames et d'honnêtes hommes des Salons» (p. 31), et la Préface, grand texte doctrinaire, d'inspiration déjà classique, sur le genre romanesque, est nettement soulignée.

6 A. Arrigoni a transcrit le texte de l'édition de Rouen, 1665, d'un accès plus facile, à la BnF, que celle de 1641-1644. Elle a procédé à une comparaison attentive des deux textes sans trouver de variantes significatives.

7 Les principes suivis pour l'établissement du texte sont indiqués dans le détail : le désir de conserver le texte en l'état, de manière à en transmettre «la saveur " (p. 55), est bienvenu. Il me semble cependant que cette fidélité au texte aurait pu trouver sa limite dans le souci de ne pas déconcerter le lecteur: si les graphies moy, luy, sont attendues, simplement parce qu'elles sont d'époque (et non parce que le $y$ est "plus clair et typographiquement plus élégant »- p. 54 - que le $i$ ), la terminaison en é des verbes du premier groupe à la première personne du passé simple (je m'embarqué) était si peu usitée qu'elle surprend: n'aurait-elle pu être révisée en ay, quitte à indiquer cette révision par une note? Quant à la ponctuation, je pense que le meilleur service à rendre 
au lecteur d'aujourd'hui aurait été de la moderniser systématiquement, mais ce point se discute évidemment.

8 Les notes sont faites consciencieusement, de manière un peu scolaire parfois. Elles ne sont pas toutes utiles : pourquoi fournir la définition de l'adjectif « extrême» (p. 111) ou du mot « port » (p. 151), qui sont toujours dans la langue, avec le sens qu'ils ont ici ? Il aurait mieux valu s'arrêter aux «isles de l'Archipelague (p. 324 et 577), c'est-à-dire celles de la mer Égée (Ægeopelagus), les Cyclades.

9 La bibliographie placée à la fin du second volume permettait de faire l'économie de celle qui se trouve p. 58-64 du premier volume, dans la section «Établissement du texte ». Commode à consulter par son classement, elle l'est moins du fait de l'usage de l'italique à la fois pour les titres des ouvrages (selon l'usage) et pour ceux des articles. Beaucoup des études répertoriées sont utiles (quelques-unes cependant ont vieilli), tandis que la présence de quelques autres est incongrue dans une édition savante (le Petit Robert, qui voisine sans vergogne avec le Dictionnaire de Moreri...). Il est vraisemblable que du temps s'est écoulé entre le dépôt du manuscrit chez les éditeurs et la sortie de l'ouvrage, ce qui explique l'absence de quelques publications récentes: ainsi l'édition de la Clélie par Ch. Morlet-Chantalat, chez Champion, dont les deux premières parties datent de 2001 et 2002 (les troisième et quatrième sont sorties en 2003 et 2004), et La Promenade de Versailles par Marie-Gabrielle Lallemand, chez le même éditeur, en 2002. Mais l'outil mis ici à la disposition des chercheurs n'en reste pas moins de premier ordre. Les fiches historiques rendent elles aussi des services, surtout lorsqu'elles portent sur des personnages mal connus (critère évidemment subjectif, variable selon les lecteurs, mais on ne peut espérer que l'homme de la rue lira Ibrahim, et pour les autres, Charles-Quint n'a pas besoin d'être présenté).

Bien que la transcription du texte ait été faite avec soin, il y reste des fautes. Il se peut que certaines figurent dans l'édition du XVII siècle (il aurait fallu les corriger); d'autres semblent bien être le fait de l'éditrice moderne. Quelques exemples : p. 331, l. $2:$ : s'il se vouloit se rendre » pour «s'il se vouloit rendre »;1. $28:$ «...Soliman, qui sans doute n'eut pas de joye... » pour « ... n'eut pas moins de joye». P. 337, 1. 25 : « ...a fin qu'on ne s'estonnait» pour «qu'on ne s'estonnast»; p. 557, 4 l. avant la fin : «quoy qu'elle fit semblable» pour «quoy qu'elle fit semblant»; p. 561, 1. 10 : «ce qui ne luy donna pas de joye » pour « ce qui ne luy donna pas peu de joye ». Certaines fautes sont bénignes, tandis que d'autres altèrent le sens du texte; si cet ouvrage connaît une seconde édition, il suffira d'une relecture pour les éliminer.

11 Mais je m'en voudrais de terminer sur cette note critique, d'autant que ce genre de problème se rencontre presque inévitablement dans la réédition de textes longs : les qualités, l'utilité de ce travail, sont évidentes. Grâce à Rosa Galli Pellegrini et à Antonella Arrigoni, voici encore un roman long du Grand Siècle remis dans le circuit de la lecture et des études - alors que l'on attend encore une édition critique de L'Astrée et qu'à mon grand regret celle du Polexandre, faite par Michel Serville pour sa thèse de doctorat, n'a pas été publiée à ce jour. On peut en être reconnaissant à nos deux collègues, non sans saluer la part croissante que prennent les chercheurs italiens dans les études dixseptiémistes. 